\title{
Title: Parallel Benefit/Cost Models for Improving Accessibility for Persons with Disabilities
}

\author{
Author: James I. Caldwell, Ph.D. \\ Systems Manufacturing Engineering \\ IBM Corporation \\ 11400 Burnet Road \\ Austin, TX 78758 \\ Tel: $\quad 512 \quad 823-8184$
}

\section{Introduction}

This paper describes a pair of analytic hierarchical models designed to estimate deltas in benefit/cost ratios as resources are recommitted within and between projects. They are utilized at a major university to distribute limited resources to maximize improvements in accessibility for persons with disabilities.

The technique of utilizing two parallel models, one for benefits and one for resource distribution, provides not only a well organized view of a large number of project details for management and executive review, but a useful working tool for staff planning and alternate resource distribution strategy studies.

\section{Background}

Early in 1988, the author was granted a Faculty Loan assignment by the International Business Machines Corporation to The University of Texas at Austin. A condition of these assignments is that the recipient work with or on behalf of a minority group. Having the personal experience of being both blind and paraplegic for the last 25 years, the author selected persons with disabilities as the most appropriate group.

Initially working directly with faculty, staff and students with disabilities within the office of the Dean of students, the author soon developed a course entitled "Human Resources, Perspectives in Ability". The course was taught within the Graduate School of Business to a cross disciplinary student population. It was well received. The classes consisted mainly of symposia presented by experts in many fields related to disability. These experts ranged from physicians and nurses specializing in physical medicine, optometry, otology, rehabilitation nursing, vocational rehabilitation counseling, etc., to people with disabilities discussing real life as lived with a physical disability. As a semester project, the students were required to organize into groups, with each group including a social science major, a computer science/engineering major and a business major. Each group was asked to choose some aspect of disability, to research the situation's background and to develop a solution strategy for the problem. These solutions were to be presented to and critiqued by a group of experts in the field selected. 
Parallel Benefit/Cost Models for Improving Accessibility ...

While the student groups were considering topics for their class projects, William H. Cunningham, President of the University, discussed with the author a major problem the University was trying to solve. The Austin campus of The University of Texas is an historic site set on relatively hilly terrain. president Cunningham was committed to making the campus and the education the University provides accessible to all people including people with disabilities. However, he expressed concern over how best to distribute scarce resources to provide the most access as rapidly as possible. Willing to accept the challenge, the author did wonder how, on a campus of almost 60,000 people, he could accomplish the task with the very limited resources at hand. Happily, there were eleven graduate students at hand, already organized into groups searching for projects. When the author described the situation to the class, they eagerly took up the challenge. Each group selected a portion of the overall access problem. One group worked on the architectural barriers on campus, one explored informational barriers (particularly in libraries), and the third studied adaptive strategies to make the University's extensive computer system accessible to persons with disabilities.

Because of the nature of the task undertaken for President Cunningham, all three groups were required to conclude their studies with action recommendations and methodology for distributing scarce resources to best accomplish their goals. Not surprisingly, three quite different models of the selected areas were developed. The situations were well researched and, finally, presented successfully to groups of professionals in the fields. Much real campus data had been gathered and three models had been developed, each well founded on searches of the literature. However, none was adequate to solve the architectural barrier problem.

The author consulted with Dr. James S. Dyer, Chairman of the Department of Management Science and Information Systems, as well. as other colleagues in the management science, architecture, engineering and rehabilitation fields to try to determine a comprehensive modeling strategy. The analytic hierarchic method was selected. This method provides an excellent modality for prioritizing projects. The EXPERT CHOICE software was selected as an excellent implementation of the method. It was possible to study a large number of projects at once, using the EXPERT CHOICE utility program "RATINGS". All diagrams of both models presented in this paper were generated using this software and are published with the kind permission of the copyright holder.

\section{The Problem}

on the surface, it would seem trivial to survey a building and determine where the architectural barriers for persons with disabilities are. standards and codes exist. certainly, students with disabilities whose education and daily lives are affected by these barriers should form a willing and expert cadre to perform surveys. There are, however, several problẹs with 
using this group as surveyors. In the first place, what is an insurmountable barrier. for one disability presents no problem at all for another. Even within a given disability group, one person will not be as handicapped as another by a given barrier. Additionally, a barrier is only a barrier if it impacts some function a person wishes to perform. Not being able to ride a horse is not a barrier for the author, since he never wished to ride a horse even when it was a physical possibility! Nevertheless, people with disabilities still constitute the most available and appropriate group to survey barriers. They have historically been utilized to survey "accessibility". The reversal of thinking from looking for access to looking for barriers requires some guidance, but is achievable.

There is one further consideration when developing a barrier model for use with young and active people with disabilities as data gatherers. Not all barriers are absolute barriers, and pride is a wonderful and powerful thing. Most students with disabilities are justifiabiy proud of the ingenious "workarounds" they have developed to cope with barriers, whether social, informational or architectural. Any model designed for this situation must not require the surveyed to define the degree or level of "barrierness" presented by. any given situation. The format must require the surveyed only to decide whether the subject area requires attention or not. The level of attention must be determined by other means.

The Model

With the analytic hierarchic method selected as a modeling technique, the first choice to be made was the survey unit. The entire campus, or even large regions of the campus, were far too large to be described within the bounds of the software chosen. To reduce the granularity to each possible barrier site would also be outside the limits of the software. A comfortable unit seemed to be to survey and model the campus one building at a time. Each was already uniquely identified numerically. Access within a building is well described in standards and codes as well as the general literature. Even the order in which the building surveys should be accomplished became relatively easy. Assuming students with disabilities to be more or less evenly distributed within the general student population, buildings utilized by the largest student populations came high on the survey list. Buildings in which few students, if any, would be likely to wish to enter were considered infeasible and eliminated from the survey for the time being. Additionally, by including the grounds immediately surrounding a building, many of the other campus barrier situations such as parking, curb cuts, etc., could be easily accommodated.

The goal was divided into two paths, INTERNAL and EXTERNAL. Paths subsequent to INTERNAL detailed possible barrier sites from the entrance doors into the building. Path subsequent to EXTERNAL described barrier sites leading up to the building. These were then further divided into more detail through three 
Parallel Benefit/Cost Models for Improving Accessibility ...

more levels. Finally, only two leaves were appended to the fifth level nodes. They require no decision by the surveyor other than whether the possible barrier site being considered is completely acceptable (0.K.) or whether it requires any attention at all to be completely acceptable (N.G.). This completed the structure of the "barrierness" side of the dual model.

The structure of the cost side of the dual model was constructed with exactly the same structure except for the final leaves. These leaves are: VERY LOW ( $\$ 0$ to $\$ 999)$, LOW $(\$ 1,000$ to $\$ 4,999)$, MEDIUM $(\$ 5,000$ to $\$ 9,999)$, HIGH $(\$ 10,000$ to $\$ 99,999)$ and VRY HIGH ( $\$ 100,000$ and up).

The next step was to set priorities within the structure. Blank priority forms were printed using the "IMPORTANCE" selection from the barrier side of the dual model. The choices were reviewed with students with disabilities and other interested people. After reaching a consensus, priorities were entered. The cost side of the dual model presented much less of a problem since dollars are dollars no matter where they are spent. AII priorities were left equal within nodes on the same branch in the cost model. The "STRUCTURE" option was used on the cost side to prevent any diluting of dollar values due to structure. Priorities for both models are shown in Figures 1 and 2 .

The dual models operate as follows:

1. Survey feasible buildings and their surrounds using a blank print-out of the next-to-bottom nodes as possible barrier sites.

2. Enter this data into the barrier model. If the potential barrier site needs any attention at all, a "1" is entered under "N.G." and a "O" under "O.K.". The process is, of course, reversed if the site needs no attention.

3. The UT Physical Plant staff is provided a list of these barrier sites surveyed as "N.G.". They then are asked to estimate the probable cost of bringing the barrier sites up to specification.

4. These estimated costs are entered into the cost model by placing a "1" in the appropriate dollar range; leaving the remainder "0".

5. Both models are synthesized. The ratings are normalized and sorted in ascending order.

It then remains to select a building which is high on the "barrier" rating list and low on the cost list. With the rich detail provided, it is simple to locate the least costly barrier site within the selected building whose elimination would provide a large increase in accessibility. Knowing the location of 
Parallel Benefit/Cost Models for Improving Accessibility ...

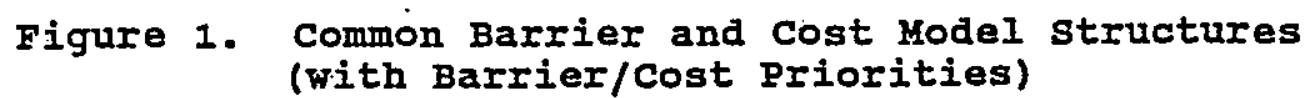

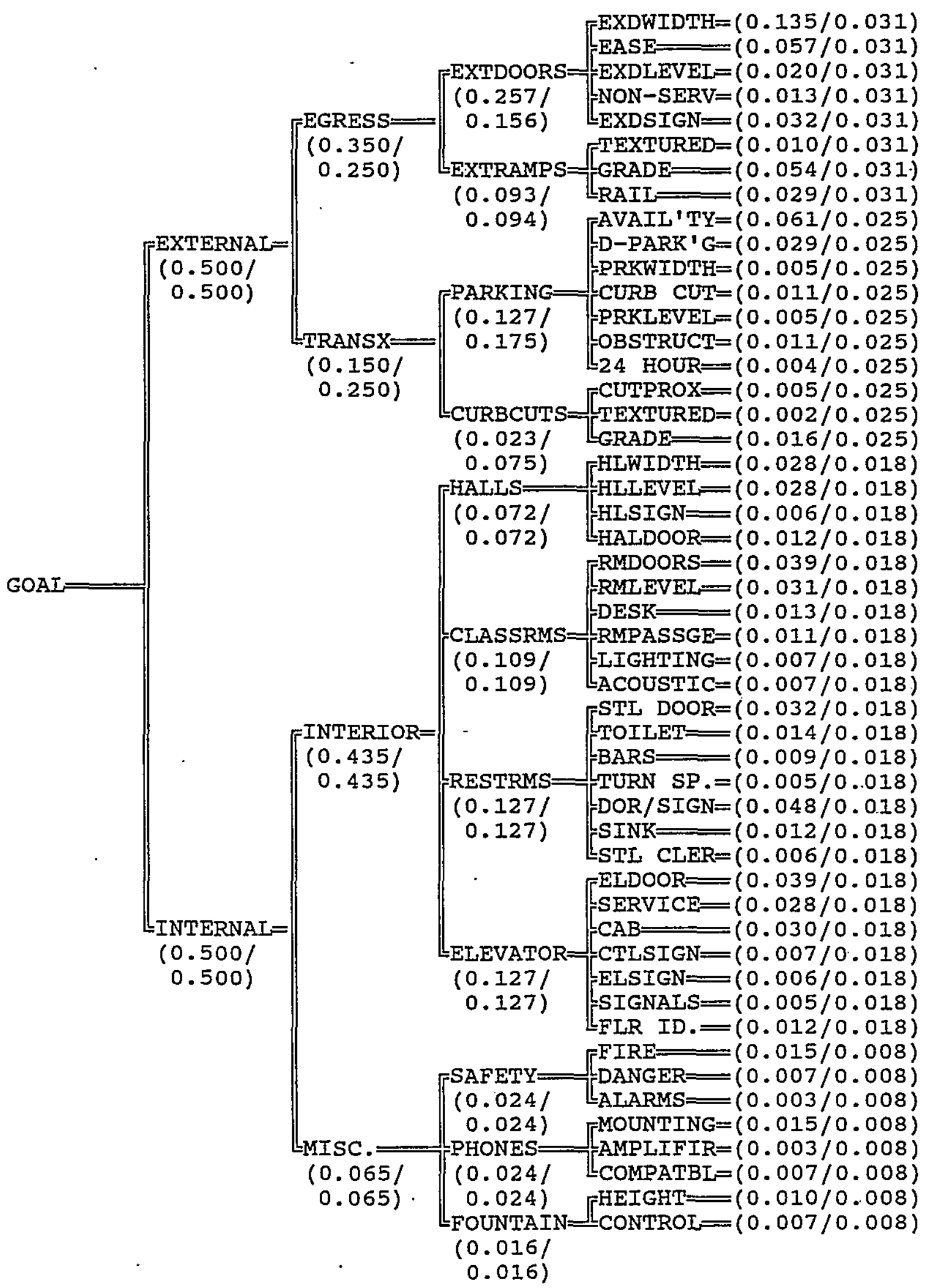


Parallel Benefit/Cost Models for Improving Accessibility ...

Figure 2. Barrier and Cost Model Lowest Leaves (with Priorities)

Barrier Lowest Leaves

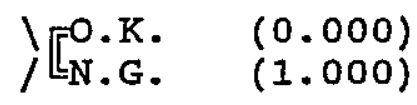

Cost Lowest Leaves

$\begin{array}{ll}\text { VERY LOW } & (0.039) \\ \text { LOW } & (0.048) \\ \text { MEDIUM } & (0.096) \\ \text { HIGH } & (0.217) \\ \text { VRY HIGH } & (0.600)\end{array}$

campus buildings and other outdoor features, there is great opportunity for engineers to maximize the use of equipment brought to a site by selecting several barrier sites requiring similar construction materials and skills for simultaneous upgrading.

The node definitions for the dual Barrier and Cost Models are:

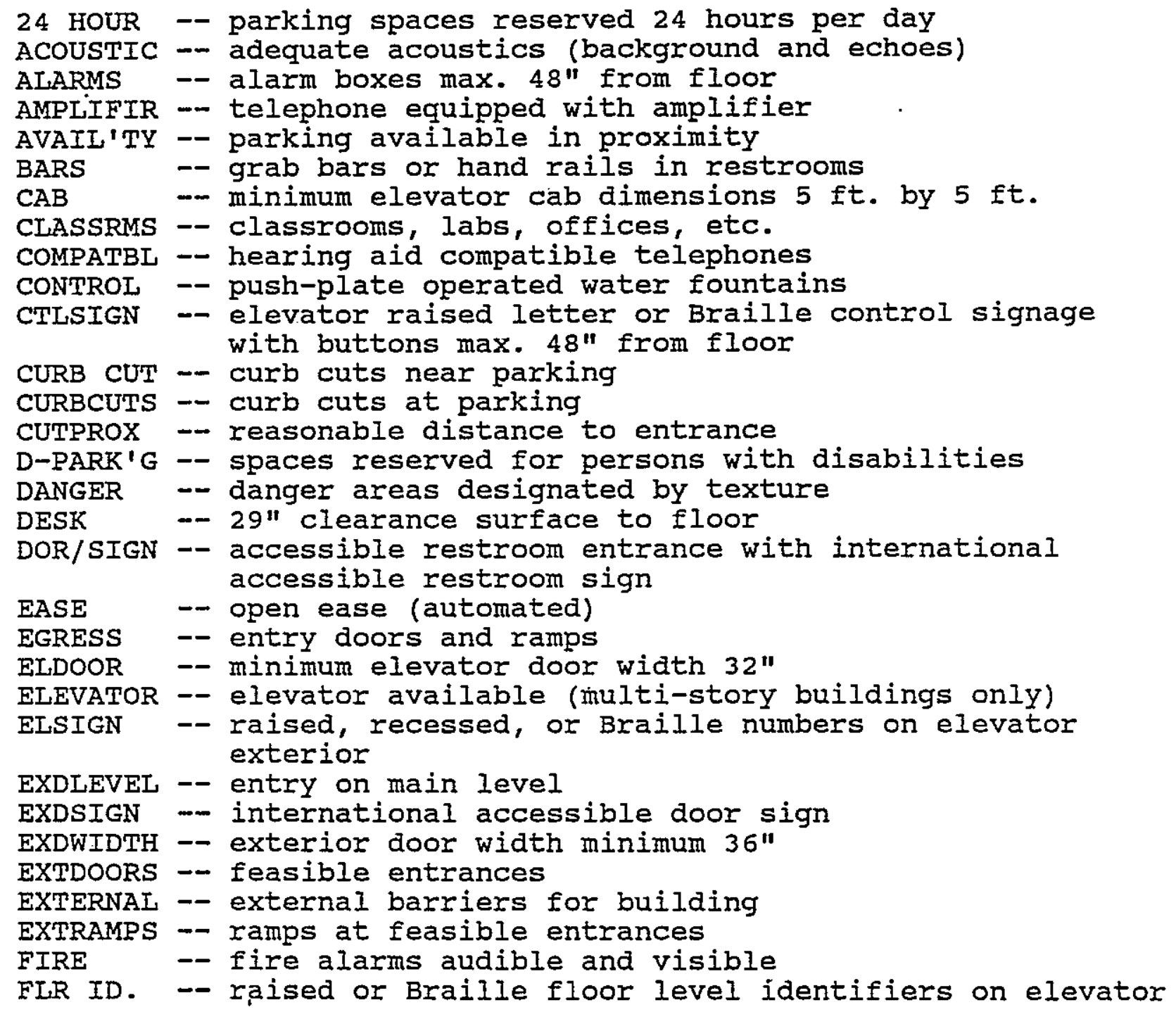


Parallel Benefit/Cost Models for Improving Accessibility ...

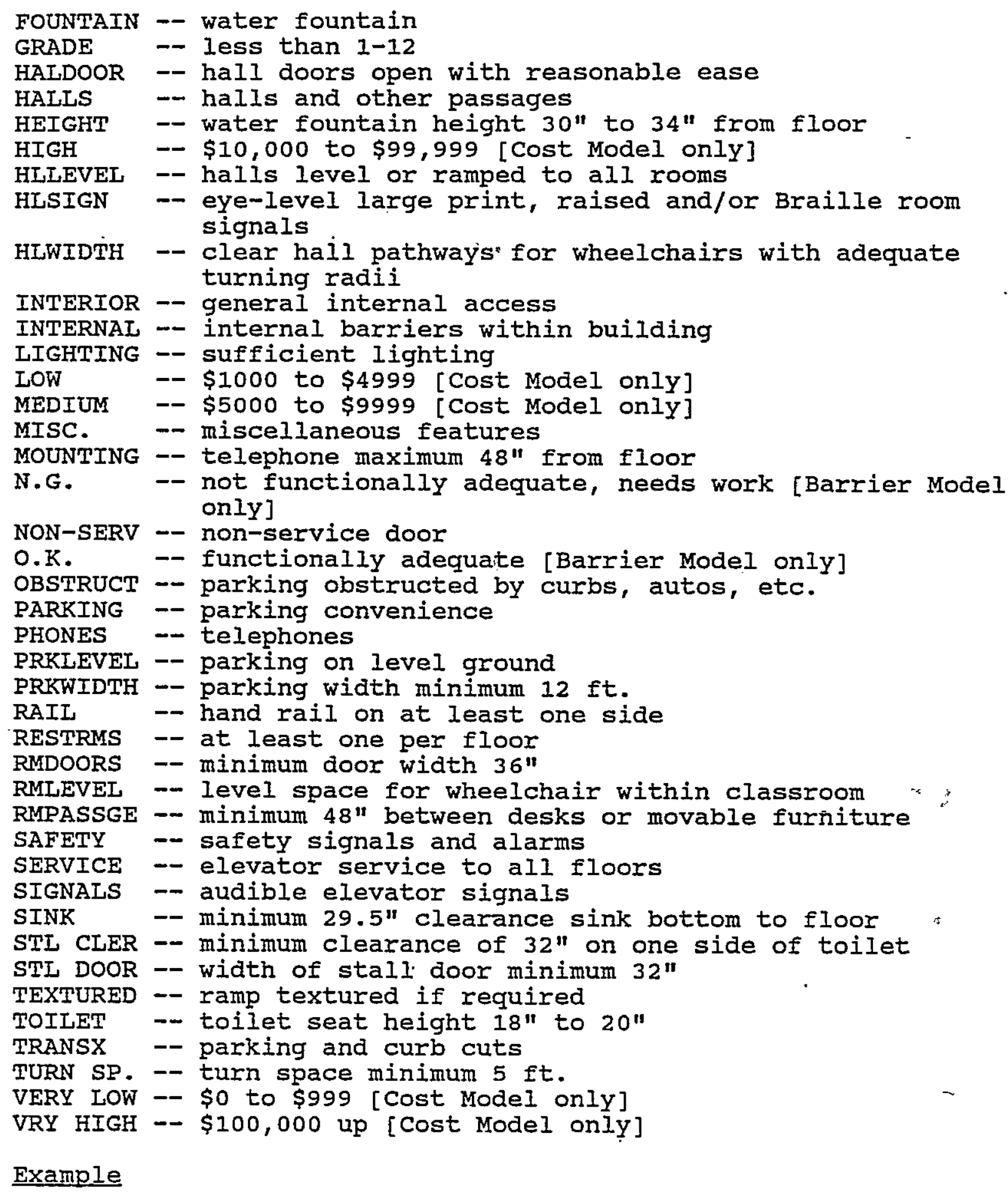

Example

An example of the use of these models is presented in Figures 3 and 4 . Three buildings have been surveyed and the barrier data has been entered in the barrier model, as shown in Figure 3 . The Physical Plant staff then estimated the costs to eliminate each of these barriers. The cost estimates were then entered in the cost model and are shown in Figure 4. The barrier ratings shown in Figure 3 show Building 1 to be the least accessible, with a total barrier rating of 0.372 . Building 1 , however, is second in. 
Parallel Benefit/Cost Models for. Improving Accessibility ...

Figure 3. Sample Barrier Model Application

\begin{tabular}{|c|c|c|c|c|c|c|c|}
\hline & EXDWIDTH & EASE & EXDLEVEL & NON-SERV & EXDSIGN & TEXTURED & RAD \\
\hline ALTERNATIVES & .1352 & .0568 & .0201 & .0130 & .0322 & .0101 & \\
\hline 1 BLDG 1 & N.G. & O.K. & N.G. & O.K. & N.G. & O.K. & \\
\hline 2 BLDG 3 & N.G. & N.G. & $0 . K$. & O.K. & N.G. & O.K. & \\
\hline
\end{tabular}

RAII AVAIL'TY D-PARK'G PRKWIDTH CURB CUT PRKLEVEL OBSTRUCT

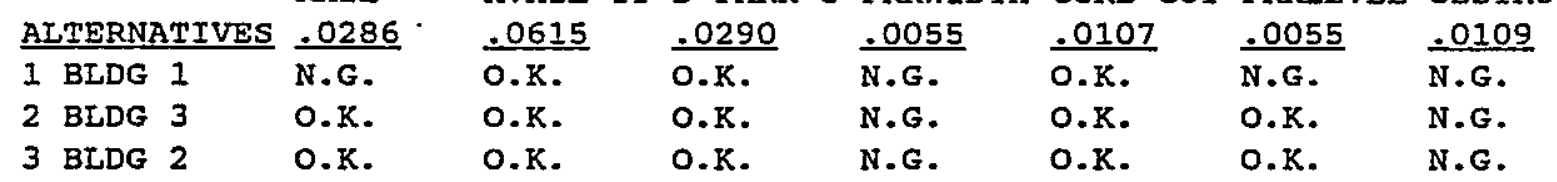

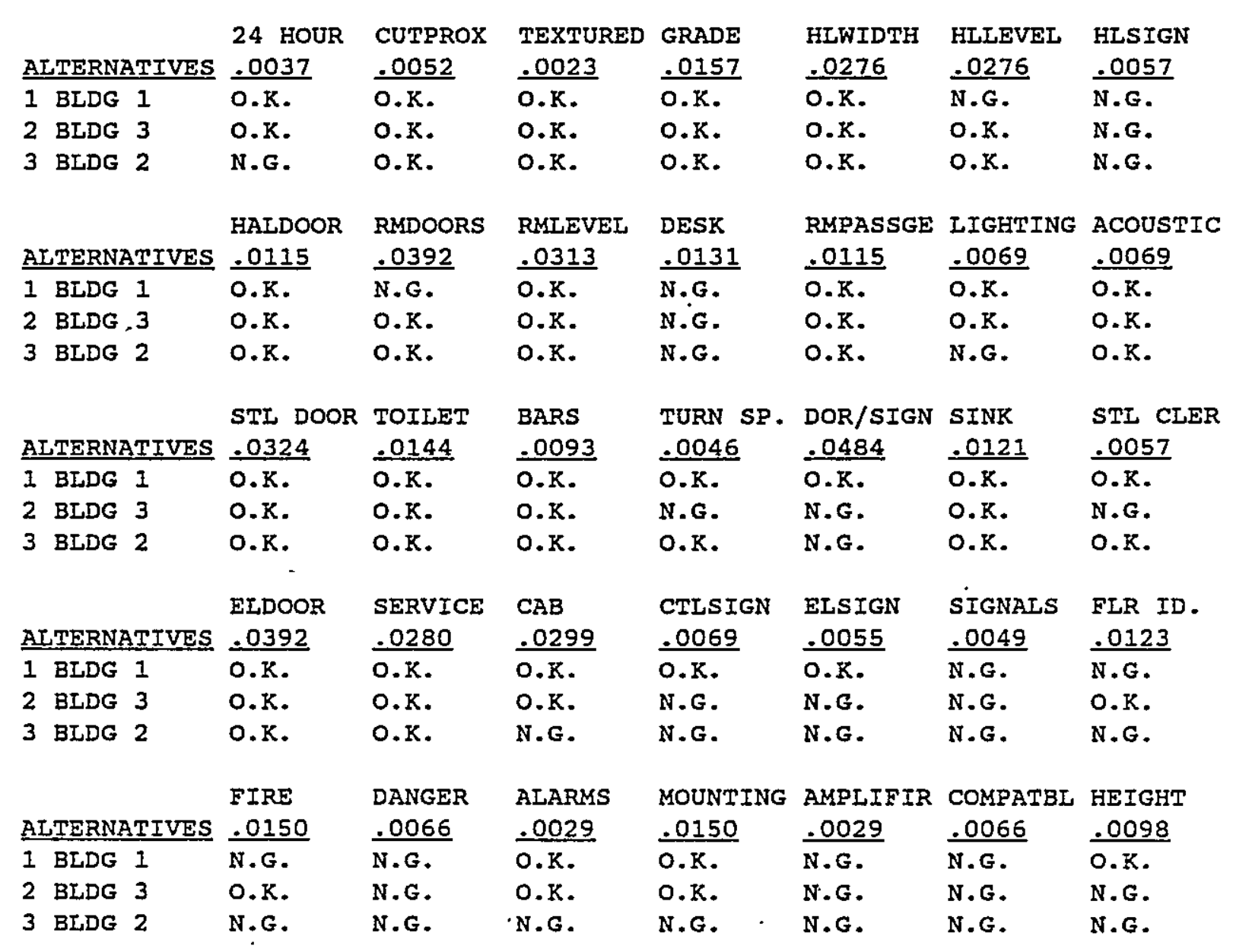

CONTROL

\begin{tabular}{|c|c|c|}
\hline ALTERNATIVES & .0065 & TOTAI \\
\hline 1 BLDG 1 & O.K. & 0.372 \\
\hline 2 BLDG 3 & N.G. & 0.368 \\
\hline 3 BLDG 2 & N.G. & 0.284 \\
\hline
\end{tabular}


Parallel Benefit/Cost Models for Improving Accessibility ...

\section{Figure 4. Sample cost Model Application}

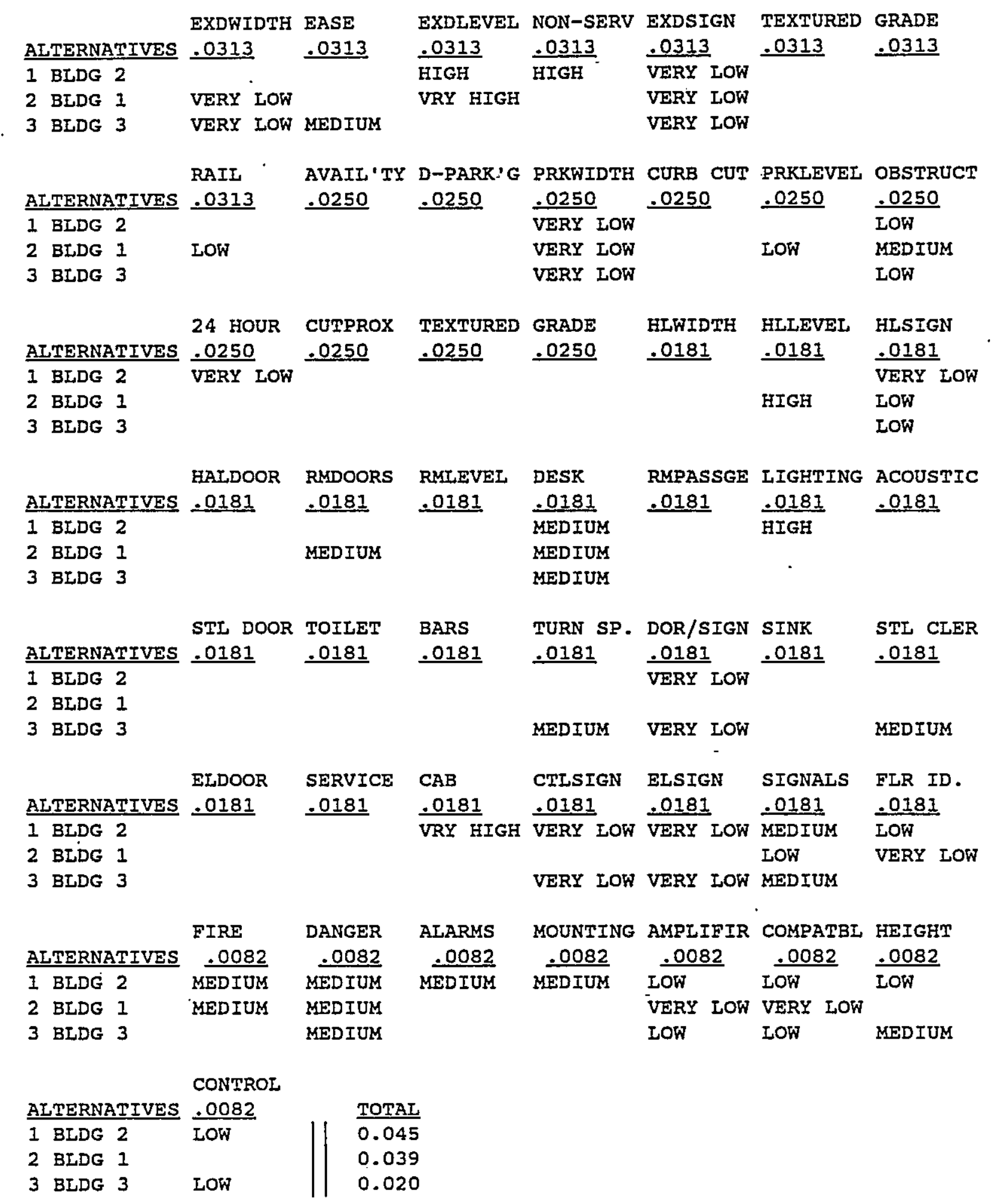


Parallel Benefit/Cost Models for Improving Accessibility ...

the ranked cost ratings, with a total cost rating of 0.039 . This combination makes Building 1 a possible candidate for accessibility improvements.

closer study of the ratings presented in the barrier model (Figure 3) show the greatest contributor to Building I's high barrier rating to be the main entrances, with a rating of 0.1352 . Details of the cost ratings (Figure 4) show the estimated cost of improvement to be "VERY LOW". This is quite often the case, perhaps only requiring an anchored bench or a large planter to be moved to provide accessibility to the building. The work is accomplished, either as a "what if" study or in fact. The rating of the main entrances of Building 1 is altered from "N.G." to "O.K." and the parallel cost estimate set to zero. Both models are resynthesized and sorted. The results of the barrier alterations are as follows: Building 1 now drops to the bottom of the total barrier ratings, with a value of 0.237 ; Building 1 remains as second in the total cost ratings, with a value of 0.038 . Thus, we achieve the best overall improvement in accessibility in the buildings surveyed for the lowest cost.

The final step was to implement the use of the models within the University. A significant amount of data had already been collected by the "Human Resources". students in the process of completing their class projects. University representatives were enthusiastic at the demonstration. An additional copy of EXPERT CHOICE was ordered for use by the Physical Plant staff. The student organization representing students with disabilities were contacted for help as surveyors and many volunteered to assist in completing the survey.

\section{Summary}

The use of a parallel pair of hierarchic models using the rating utility to document a large number of projects has two very distinct advantages. First, they provide an excellent platform for documenting a large number of details within each project. The barrier ratings presented in figure 3 show graphically the barrier sites within each building. Similarly, the estimated expense for removing each barrier is simple to find in the paraliel cost ratings shown in Figure 4 .

Second, the models provide the tools to determine which building is the best candidate for improvement from a barrier/cost standpoint. The effect of a selected improvement may be simulated before actual work begins. More generally, the effect of a large improvement project involving eliminating barrier sites within one building or across several buildings may be similarly studied.

With the increasing demand for improvements to accessibility for persons with disabilities demanded by both public conscience and recently enacted public law (e.g., Americans with Disabilities Act, July 1990), the conservation of resources committed to these projects is now more important than ever. 
Parallel Benefit/Cost Models for Improping Accessibility ...

\section{References}

EXPERT CHOICE (c), Decision Support Software, Inc., 1300 Vincent place, McLean, VA 22101. Version BE7.0 was used in this project.

American National Standard A117.1-1986, "Buildings and Facilities - Providing Accessibility and Usability for Physically Handicapped People," published by American National Standards Institute, Inc., 1430 Broadway', New York, NY 10018.

Uniform Building code, 1988, published by International Conference of Building officials, Whittier, California. 